\title{
Optical fringe multiplication technique with TEM nano-moiré method
}

\author{
Huimin Xie ${ }^{1}$, Zhanwei Liu ${ }^{1}$, Daining Fang ${ }^{1}$, Fulong Dai ${ }^{1}$, \\ Yongming $\mathrm{Xing}^{2}$ and Yapu Zhao ${ }^{3}$ \\ ${ }^{1}$ Department of Engineering Mechanics, FML, Tsinghua University, 100084 Beijing, \\ People's Republic of China \\ ${ }^{2}$ Department of Engineering Mechanics, Inner Mongolia Polytechnic University, \\ 010062 Hohhot, People's Republic of China \\ ${ }^{3}$ LNM, Institute of Mechanics, Chinese Academy of Science, 100080 Beijing, \\ People's Republic of China \\ E-mail: liuzw01@mails.tsinghua.edu.cn
}

Received 24 July 2004, in final form 13 October 2004

Published 21 January 2005

Online at stacks.iop.org/MST/16/529

\begin{abstract}
In this paper, a nano-moiré fringe multiplication method is proposed, which can be used to measure nano-deformation of single crystal materials. The lattice structure of $\mathrm{Si}$ (111) is recorded on a film at a given magnification under a transmission microscope, which acts as a specimen grating. A parallel grating (binary type) on glass or film is selected as a reference grating. A multiplied nano-moiré fringe pattern can be reproduced in a $4 f$ optical filter system with the specimen grating and the prepared reference grating. The successful results illustrate that this method can be used to measure deformation in nanometre scale. The method is especially useful in the measurement of the inhomogeneous displacement field, and can be utilized to characterize nano-mechanical behaviour of materials such as dislocation and atomic bond failure.
\end{abstract}

Keywords: nano-moiré, fringe multiplication, crystal lattice

\section{Introduction}

The ordinary moiré method was introduced by Weller in 1948 [1] and has been improved by many researchers [2]. The sensitivity of displacement measurement with this method depends upon the frequency of the applied grating. In the measurement of the ordinary moire method, the applicable grating frequency is generally lower than 100 lines $\mathrm{mm}^{-1}$, thus this method can only be used to measure large deformation. With the development of techniques for producing holographic gratings, moiré interferometry has been widely used in experimental stress analysis and applied to the field of mesomechanics since the 1970s [3, 4]. In the measurement of moiré interferometry, the frequency of the applicable specimen grating has a range from 600 lines $\mathrm{mm}^{-1}$ to 2400 lines $\mathrm{mm}^{-1}$. In the 1990s, Kishimoto [5] proposed the electron beam moiré techniques, and the moiré grating was fabricated by electron beam lithography. From the available reports, a specimen grating up to the frequency of 10000 lines $\mathrm{mm}^{-1}$ can be utilized in the electron beam moiré measurement. In 1993,
Read [6] proposed the scanning moiré method. In his study, the scanning lines in a SEM monitor or CCD video camera were used as a reference grating to form scanning moiré fringes. This method could provide an easy and effective way to improve spatial resolution in the moiré measurement.

The mechanical behaviour of engineering materials in the nanoscopic range have drawn the attention of researchers on materials and mechanics in recent years. In order to understand the rules of this behaviour, deformation measurement techniques with a sensitivity and spatial resolution at nanometre level are required [7, 8]. However, the above-mentioned methods obviously cannot meet such requirements.

Hashimoto and Ueda [9] and Pashley [10] observed dislocation by means of moiré pattern on electron micrographs in 1957. In their works, a crystal lattice was first introduced to serve as a grating. In 1999, Dai and Xing proposed a nano-moiré method with a transmission electron microscope (TEM) image [11]. In their study, a crystal lattice acted as a specimen grating and a unidirectional coarse grating as a 
reference grating. The two gratings are superposed, and a moiré pattern in nano-scale can be generated. Xie et al [12] developed the atomic force microscope (AFM) scanning moiré method. In his study, the AFM moiré pattern is formed by AFM probe scanning lines and an atomic lattice.

In order to improve the accuracy of the moiré measurement, some efforts were made to multiply moiré fringes. A typical example is the super high-sensitivity moiré interferometry with holographic wave-front warpage multiplication [13]. Another example is the optical fringe multiplication in moiré interferometry [14].

High resolution TEM has been extensively applied to observe the structure features in nano-scale. It has been applied to the examination of particle morphologies and size distributions, to analyse structural features of intracellular magnetite particles [15], to observe $\Sigma=3$ and $\Sigma=9$ tilt about the $\langle 011\rangle$ axis (first- and second-order twin) boundaries in a specimen cleaved from a melt grown CdTe bulk crystal [16].

In this paper, an optical fringe multiplication technique with a TEM nano-moiré method is presented. This method can be used to measure in-plane displacement and strain in nanometre scale. Especially, it is suitable to reveal nanomechanical behaviour such as dislocation and atomic bond failure.

\section{Fringe multiplication principle of TEM nano-moiré method}

\subsection{Formation mechanism of TEM nano-moiré fringes}

In terms of the theory of material physics, a single crystal has a periodic structure, which consists of atoms and molecules. Thus, it can be considered as a nano-grating with high frequency, which contains several millions of lines per millimetre. In the measurement, the reference grating is prepared with a frequency of a few tens of lines per millimetre.

In order to form TEM nano-moiré fringes, the specimen is recorded on a photosensitive film before and after loading. The films are utilized as specimen gratings. A TEM (the type is JEM-2010F) with atomic resolution is used to acquire images of the sample. Compared with AFM and STM (scanning tunnelling microscope) nano-moiré methods [17], this method can provide a larger observation field and better contrast of moiré fringes.

In this study, $\mathrm{Si}$ (111) is selected as the measured object. A TEM image of the lattice structure of Si (111) is shown in figure 1. The period of this lattice is $0.304 \mathrm{~nm}$, which represents a grating with a frequency of 3289474 lines $\mathrm{mm}^{-1}$.

Before conducting a TEM nano-moiré test, the frequency of a specimen grating is calibrated. The relationship between the frequency of the specimen grating and the magnification is analysed. In the calibration, TEM images of the specimen at different magnification from X150K to $\mathrm{X} 800 \mathrm{~K}$ by an increment of $\mathrm{X} 50 \mathrm{~K}$ were collected and recorded on photosensitive films. Then these films are used to analyse the frequency of the specimen grating. Each film is clamped in the object plane 4 in a $4 f$ optical filter system [18] (as shown in figure 2). When a collimated laser beam passes through the specimen grating and the transforming lens 5, a diffraction

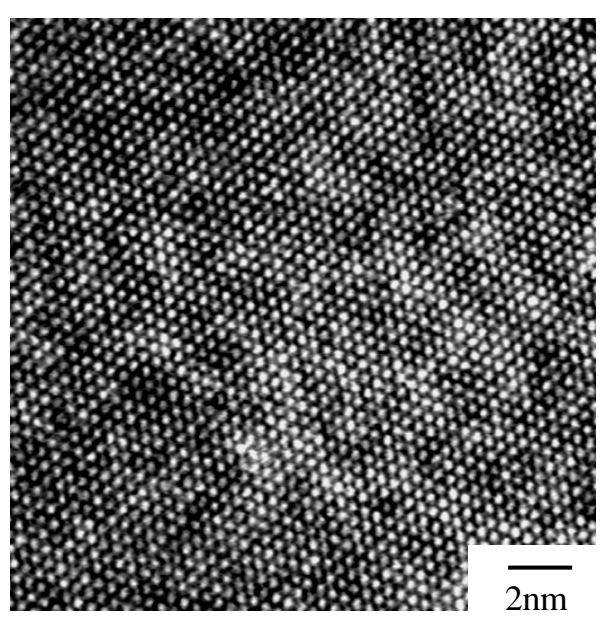

Figure 1. TEM image of Si (111).

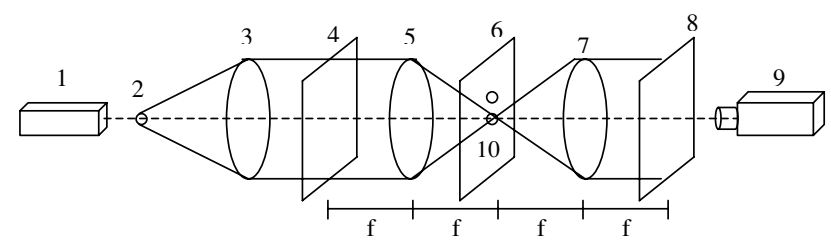

Figure 2. Schematic diagram of the $4 \mathrm{f}$ optical filter system. 1, Laser; 2, beam enlarger; 3, collimating lens; 4, object plane; 5 , transforming lens; 6 , spectrum plane; 7 , image lens; 8 , image plane; 9, CCD and computer; and 10, pinhole.

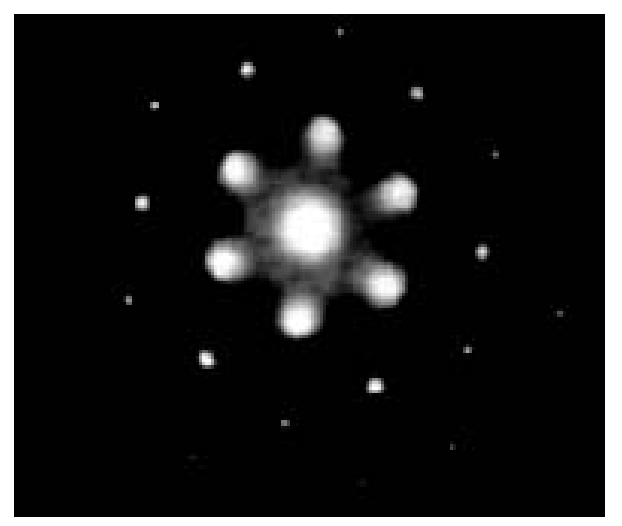

Figure 3. Diffraction spectrum of a TEM image of Si (111).

spectrum will appear on plane 6 . In the plane, it is found clearly that there are several spots of diffraction orders. (A diffraction spectrum of $\mathrm{Si}$ (111) is shown in figure 3.) From figure 3, it can be seen that there are six larger diffraction spots near the centre, which can be considered as three matched pairs. The two spots of each pair are symmetric with respect to the centre, and their corresponding diffraction orders should be defined as \pm 1 , respectively. In this study, we are especially concerned with the \pm 1 st diffraction order pairs. By selectively filtering out all but an arbitrary \pm 1 st diffraction order pair, a unidirectional grating image can be discerned in the image plane 8 . The grating image is captured by a CCD camera and can be observed on the screen of the computer 9. From the grating image, the frequency of the original specimen grating can be expressed as 


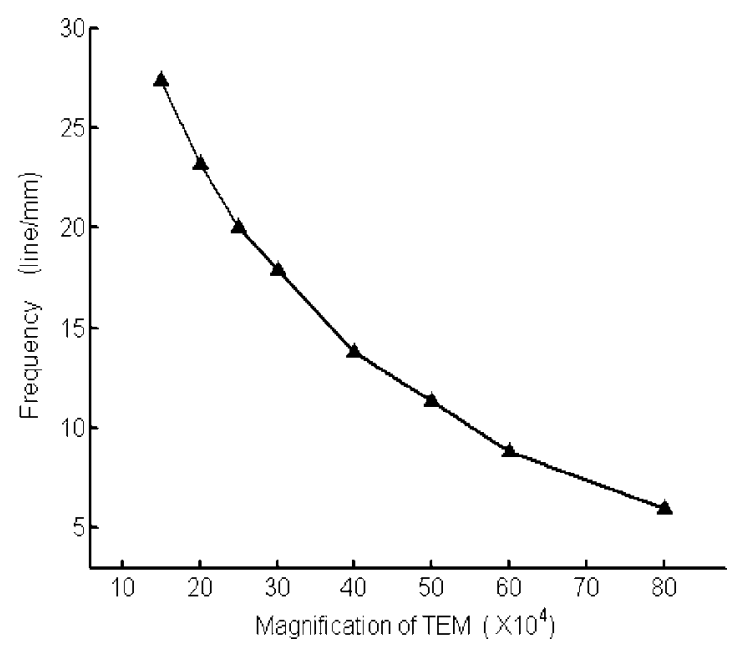

Figure 4. The relationship between the frequency of a specimen grating and the magnification of TEM.

$$
f=\frac{m-1}{2 H} M
$$

where $m$ is the number of grating lines, $H$ is the spacing of $m$ strip grating lines, $M$ is the magnification of the whole optical system. A calibration result is shown in figure 4.

In the experiment, a deformed specimen grating image can be obtained in the image plane, regardless of the \pm 1 st diffraction order pair that passes through the pinhole. If a reference grating with a matching frequency is placed in the image plane, a moiré pattern can be discerned [2]. By rotating the reference grating to an appropriate angle, a parallel moiré fringe pattern can be obtained in the image plane.

\subsection{Multiplication principle of TEM nano-moiré fringe}

In this study, a $4 f$ optical filter system is utilized to multiply TEM nano-moiré fringes. By selectively filtering out some diffraction orders, the frequency of the specimen grating can be multiplied. In order to generate a moiré pattern, the reference grating should be determined according to the multiplied frequency of the specimen grating.

In the experiment, the specimen grating is clamped by two pieces of optical glass, which are placed in a 3D adjustable stage. A thin metal plate with a thickness of $1 \mathrm{~mm}$ is placed in the spectrum plane 6 . Two circular holes with diameter $1.5 \mathrm{~mm}$ are manufactured in the plate and they act as filter pinholes. In the plate (spectrum plane 6), there are several diffraction order pairs. When the $\pm n$th order diffraction beams are selected to pass through the pinholes, a specimen grating with multiplied frequency can be discerned in the image plane. When the multiplied specimen grating is superimposed by a unidirectional reference grating with a matched frequency, the multiplied moiré fringe pattern can be obtained and collected by a CCD camera. In this experiment, the frequency of a specimen grating for Fourier filtering should be more than 5 lines $\mathrm{mm}^{-1}$ to avoid the overlap of diffraction orders.

When a collimated laser beam travels through a deformed specimen grating, the respective transmission function

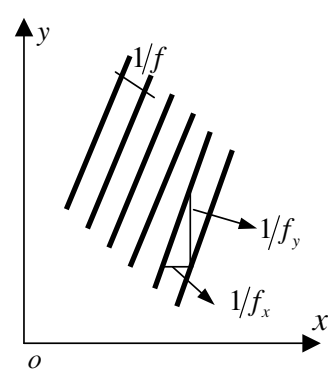

Figure 5. Schematic diagram of spatial frequency of a specimen grating.

$f_{0}(x, y)$ can be expressed by a Fourier series [14] (take a unidirectional grating for example)

$$
\begin{gathered}
f_{0}(x, y)=\sum_{n=-\infty}^{+\infty} C_{n} \exp \left[\mathrm{i} 2 n \pi\left(f_{x} x+f_{y} y\right)\right] \\
\left(f_{x}=\frac{1}{d_{1}+\delta_{1}}, f_{y}=\frac{1}{d_{2}+\delta_{2}}\right)
\end{gathered}
$$

where $C_{n}$ is a constant, $f_{x}, f_{y}$ are the frequency components of the local deformed specimen grating in the $x$ and $y$ axes (as shown in figure 5), respectively. $d_{1}, d_{2}$ are the components of the initial pitch of the specimen grating, $\delta_{1}\left(\delta_{1}=\delta_{1}(x, y)\right)$, $\delta_{2}\left(\delta_{2}=\delta_{2}(x, y)\right)$ are the components of the departure of grating lines from straightness in the $x$ and $y$ direction. In the spectrum plane, the complex amplitude function $F_{0}(\xi, \eta)$ of light waves can be expressed by the Fourier transform of the $f_{0}(x, y)$,

$F_{0}(\xi, \eta)=\iint_{-\infty}^{+\infty} f_{0}(x, y) \exp [-\mathrm{i} 2 \pi(\xi x+\eta y)] \mathrm{d} x \mathrm{~d} y$.

From equations (2) and (3), the $F_{0}(\xi, \eta)$ can be expressed as [14]

$$
F_{0}(\xi, \eta)=\sum_{n=-\infty}^{+\infty} C_{n} \delta\left(\xi-n f_{x}, \eta-n f_{y}\right)
$$

If $\xi$ is not equal to $n f_{x}$ or $\eta$ is not equal to $n f_{y}$, we have $F_{0}(\xi, \eta)=0$, it indicates that discrete diffraction spots are formed along with the different frequencies $\left(n f_{x}, n f_{y}\right)$ in the spectrum plane. If a pair of the $\pm n$th diffraction beam passes through the pinholes, the complex amplitude $E_{n}, E_{-n}$ of the corresponding light waves can be written as

$$
\begin{aligned}
& E_{n}=C_{n} \exp \left[\mathrm{i} 2 n \pi\left(f_{x} x+f_{y} y\right)\right] \\
& E_{-n}=C_{n} \exp \left[-\mathrm{i} 2 n \pi\left(f_{x} x+f_{y} y\right)\right] .
\end{aligned}
$$

In the image plane, the interference intensity distribution of the two light waves is [14]

$$
I_{ \pm n}=2 C_{n}^{2}\left[1+\cos 2 \pi\left(2 n f_{x} x+2 n f_{y} y\right)\right] .
$$

From equation (6), it can be seen that the specimen grating frequency is multiplied with a factor of $2 n$. According to [14], the displacement measurement sensitivity can be increased $2 n$ times with the specimen grating.

If the three +1 st diffraction beams are selected to pass through the pinholes successively, an original specimen grating image is captured and is as shown in figure $6(a)$. If the \pm 1 st diffraction beams of each pair pass through the pinholes in turn, the specimen grating with a multiplied frequency is 


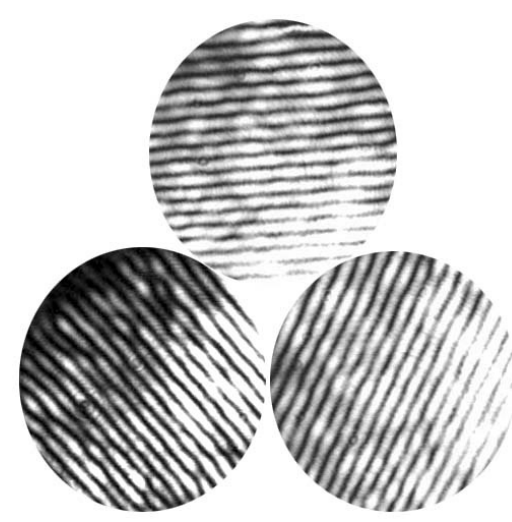

(a)

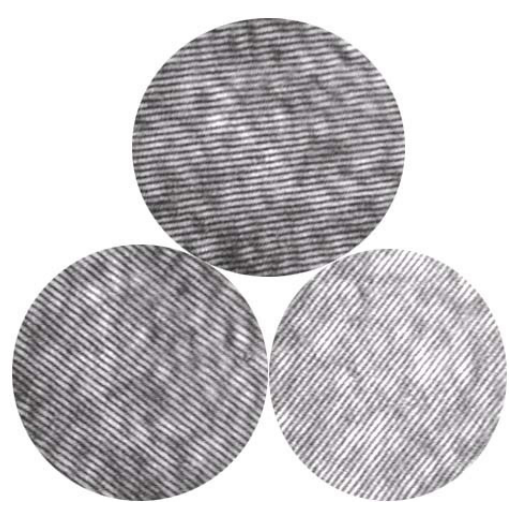

(b)

Figure 6. Specimen grating. (a) Original specimen grating. (b) Grating rosette with a frequency of multiplying times 2.

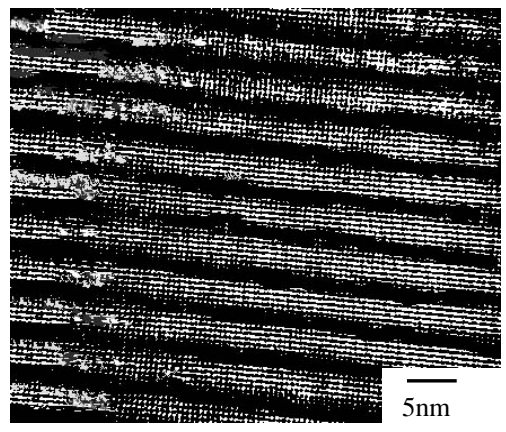

(a)

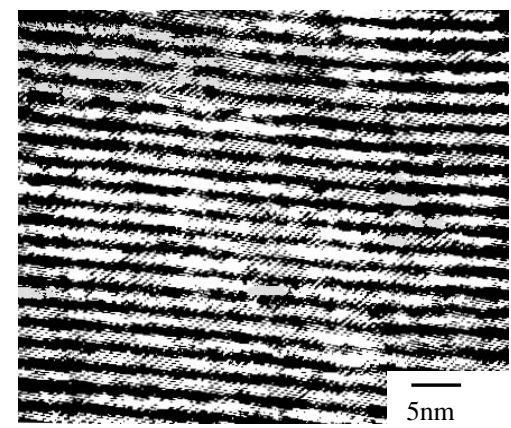

(b)

Figure 7. TEM nano-moiré fringe pattern. (a) Original carrier fringe pattern. (b) Carrier fringe pattern multiplied times 2.

as shown in figure $6(b)$. The three groups of gratings can be considered as a rosette with three different directions [19]. The intersection angle between two adjacent groups is $120^{\circ}$. Each grating in the rosette can be applied to form moiré fringes independently.

In the experiment, TEM images of $\mathrm{Si}$ (111) with a magnification of $\times 300 \mathrm{k}$ are utilized. When the original specimen grating was superimposed with a unidirectional reference grating with a frequency of 20 lines $\mathrm{mm}^{-1}$, an original carrier moiré fringe pattern could be formed and is shown in figure $7(a)$. When the specimen grating with multiplied frequency is overlapped with a unidirectional grating with a frequency of 40 lines $\mathrm{mm}^{-1}$, a multiplied moire fringe pattern with carrier is obtained and is shown in figure $7(b)$.

In the experiment, a unidirectional reference grating was placed on an adjustable stage in the image plane, which can be conveniently adjusted to generate the moiré fringes with a grating rosette. A schematic diagram of three directional moiré fringes multiplied is shown in figure 8. From figure 8 , the displacement and strain components in three different directions can be expressed respectively as [20]

$$
\begin{gathered}
u_{\theta i}=\frac{N_{i}}{2 M_{0} n f} \\
\varepsilon_{\theta i}=\frac{M}{2 n h_{i i} f} \quad\left(i=1,2,3 ; \theta_{i}=90^{\circ}, 210^{\circ}, 330^{\circ}\right)
\end{gathered}
$$

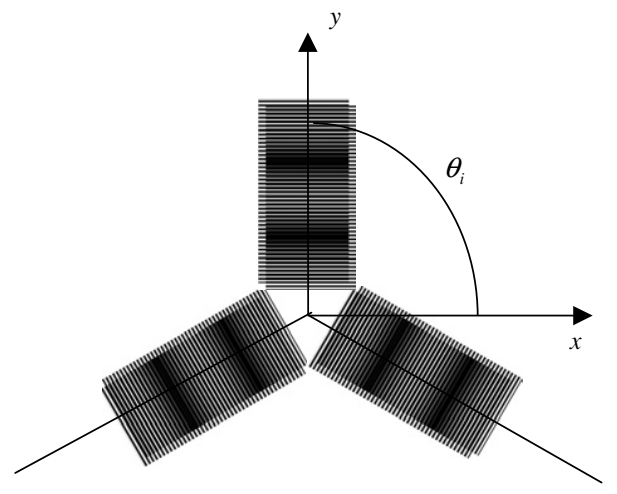

Figure 8. Schematic diagram of three directional moiré fringes.

where $n$ is the diffraction order, $M_{0}$ is the magnification of TEM, $N_{i}$ is the order of moire fringes, $h_{i i}$ is the fringe spacing in the $\theta_{i}$ direction.

The sign of the normal strain can be determined by rotating the reference grating. When the moiré fringes move in the same direction as the rotation of the reference grating, the normal strain is tensile. Otherwise, the normal strain is compressive.

The relation among the $\varepsilon_{\theta i}, \varepsilon_{x}$ and $\varepsilon_{y}$ can be expressed as [20]

$\varepsilon_{\theta i}=\frac{\varepsilon_{x}+\varepsilon_{y}}{2}+\frac{\varepsilon_{x}-\varepsilon_{y}}{2} \cos 2 \theta_{i}+\frac{\gamma_{x y}}{2} \sin 2 \theta_{i} \quad(i=1,2,3)$. 


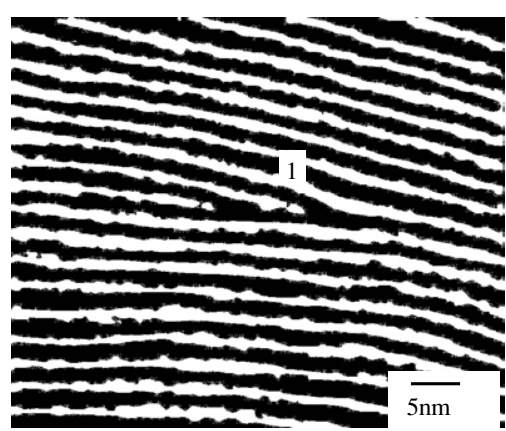

(a)

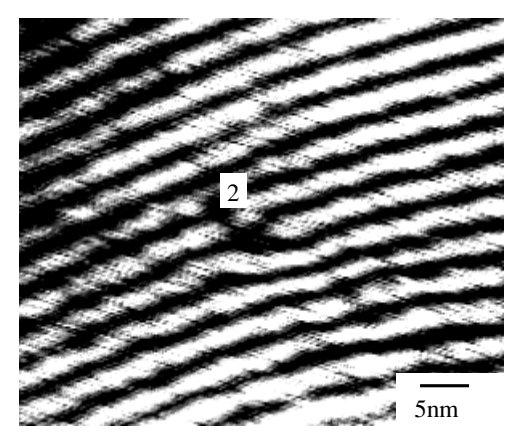

(b)

Figure 9. Fringe pattern around dislocation. (a) Fringe pattern around dislocation 1. (b) Fringe pattern around dislocation 2.

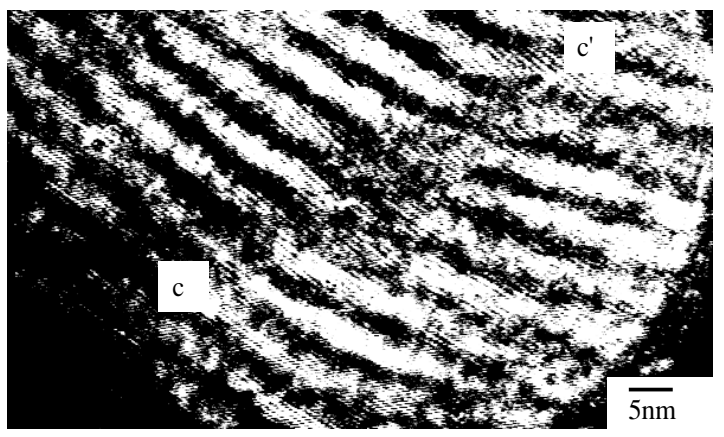

Figure 10. Fringe pattern near atomic bond failure.

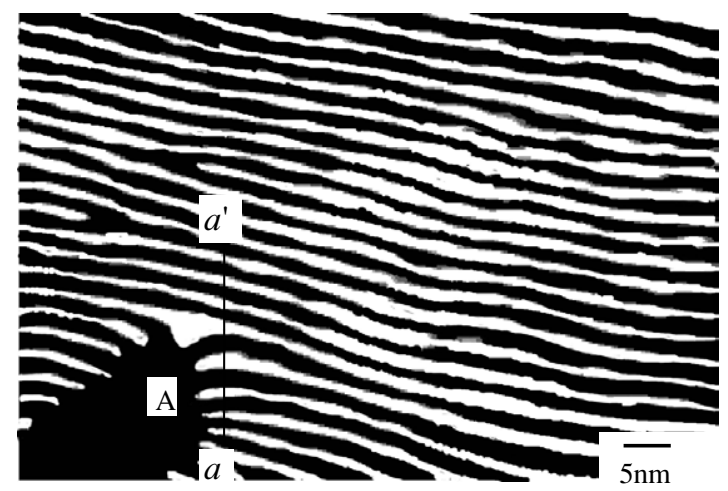

Figure 11. Fringe pattern around an inclusion.

From equations (8) and (9), the normal strain $\varepsilon_{x}, \varepsilon_{y}$ and shear strain $\gamma_{x y}$ can be easily determined.

\section{Experimental results with a multiplication factor 2}

The main cleavage surface is $\mathrm{Si}(111)$, the dislocation emission always occurs from this surface. In this experiment, the fringe joining can be easily observed (as shown at point 1 in figure $9(a)$ and point 2 in figure $9(b)$ ). The fringe joining is a signature of thread dislocations [11]. Figure 10 shows the fringe pattern near atomic bond failures along the layer $c c^{\prime}$, the fringes are sheared and have different directions along the layer. At the region A in figure 11, moiré fringes disappear. A local electron diffraction pattern verifies that they are incurred by the non-crystalline inclusion. The fringes around the

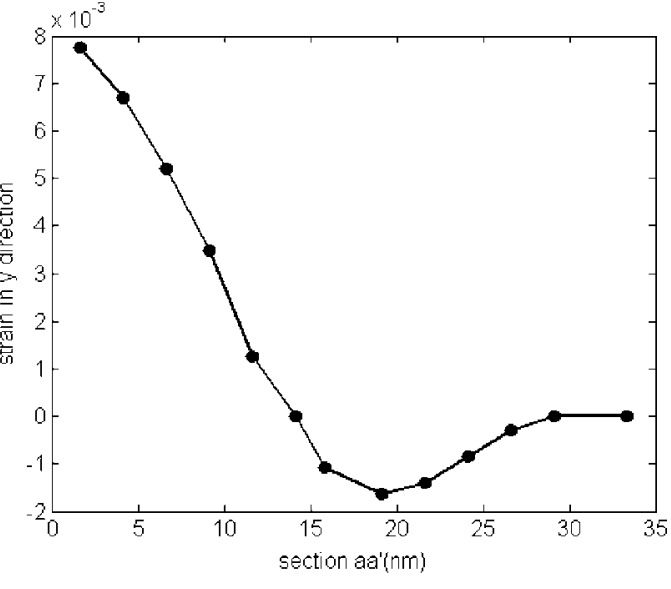

Figure 12. Strain distribution along the section $a a^{\prime}$.

inclusions become twisted. These inclusions result in nonuniform residual deformation. Figure 12 shows the residual strain distribution in the $y$ axis direction along the section $a a^{\prime}$.

\section{Conclusion}

A new technique for multiplying TEM nano-moiré fringes is developed, which can be used to improve the accuracy of the displacement measurement. This method is utilized to measure the nano-deformation around an inclusion. The successful experimental results verify the feasibility of this method.

The method can be used to measure in-plane displacement and strain in nanometre scale. Especially, it is suitable to reveal nano-mechanical behaviour such as dislocation and atomic bond failure. The method offers an effective experimental tool for nano-mechanics study, and it has a good potential for further application.

\section{Acknowledgments}

The work is supported by the National Natural Science Foundation of China (under grants 10232030, 10121202), the Key grant project of Chinese Ministry of Education (No 0306), the Research Foundation from the Tsinghua University, Opening research fund from LNM and the Projectsponsored by SRF for ROCS, SEM. The authors are especially 
grateful to Professor Wei Yang for his support and help during this research.

\section{References}

[1] Weller R and Shepard B M 1948 Displacement measurement by mechanical interferometry Proc. Soc. Exp. Stress. Anal. 6 35-8

[2] Patorski K 1993 Handbook of the Moiré Fringe Technique (Amsterdam: Elsevier)

[3] Guo M J and Xiao L X 1997 Stress measurement of composite joint with pinhole using moiré interferometry Acta Mater. Compos. Sin. 14 122-6

[4] Han B, Post D and Ifju P 2001 Moiré interferometry for engineering mechanics: current practices and future development J. Strain Anal. Eng. Des. 36 101-17

[5] Kishimoto S, Egashira M and Shinya N 1993 Micro-creep deformation measurement by a moiré method using electron beam lithography and electron beam scan $O p t$ Eng. 32 522-6

[6] Read D T, Dally J W and Szanto M 1993 Scanning moiré at high magnification using optical methods Exp. Mech. $\mathbf{3 3}$ $110-6$

[7] Xie H M, Dai F L, Yang H, Liu N, Dietz P and Schmidt A 1998 The residual deformation measurement of nanometer crack in $\mathrm{Si}$ (111) $7 \times 7$ with the scanning tunneling microscope Scanning Probe Microsc. 1 181-5

[8] Chen C J 1993 Introduction to Scanning Tunnel Microscope (New York: Oxford University Press)

[9] Hashimoto H and Ueda R 1957 Detection of dislocation by the moiré pattern in electron micrographs Acta Crystallogr. 10143
[10] Pashley D W 1957 Observation of dislocation in metals by means of moiré pattern on electron micrographs Nature 179 $752-5$

[11] Dai F L and Xing Y M 1999 Nano-moiré method Acta Mech. Sin. $15283-8$

[12] Xie H M, Kishimoto S, Asundi A, Chai G B, Shinya N, Yu J and Ngoi B K 2000 In-plane deformation measurement using the atomic force microscope moire method Nanotechnology 11 24-9

[13] Czarnek R 1990 Super high sensitivity moiré interferometry with optical multiplication Opt. Laser Eng. 13 87-98

[14] Qing X L, Qin Y W and Dai F L 1995 Optical fringe multiplication in moiré interferometry Appl. Opt. 34 $7291-4$

[15] Devouard B, Posfai M, Hua X, Bazylinski D A, Frankel R B and Buseck P R 1998 Magnetite from magnetotactic bacteria: size distributions and twining Am. Mineral. 83 1387-98

[16] Milenov T I, Dimov V I, Khaltakova N G and Gospodinov M M 2000 HRTEM observations of $\Sigma=3$ and $\Sigma=9$ tilt about $\langle 011\rangle$ axis grain boundaries in bulk CdTe crystals Cryst. Res. Technol. 35 1331-41

[17] Liu Z W, Xie H M, Fang D N and Dai F L 2004 A novel experimental technique-nano-moiré method with scanning tunneling microscope (STM) J. Mater. Process. Technol. $14877-82$

[18] Goodman J W 1968 Introduction to Fourier Optics (New York: McGraw-Hill)

[19] Chen J B, Zhou B M and Ginesu F 2001 Measurement on residual stresses by grating rosette and moiré interference Chin. J. Lasers 28 746-8

[20] Zhang R Y and Lu Y Z 1981 Experimental Stress Analysis (China: Academic) 